\title{
Questionnaire survey of thrombolytic treatment in accident and emergency departments in the United Kingdom
}

\author{
Stuart Hood, David Birnie, Lorna Swan, W Stewart Hillis
}

See $p 280$

Department of

Cardiology, Victoria

Infirmary, Glasgow

G42 9TY

Stuart Hood,

registrar

Department of

Medicine, Dumfries

and Galloway Royal

Infirmary, Dumfries

DG1 4AL

David Birnie,

registrar

University

Department of

Medicine and

Therapeutics,

Western Infirmary,

Glasgow G11 6NT

Lorna Swan,

research fellow

W Stewart Hillis,

reader

Correspondence to:

Dr S Hood,

Department of

Medical Cardiology,

Royal Infirmary,

Glasgow G31 2ER

BMJ 1998;316:274
Numerous randomised trials have shown that thrombolytic treatment reduces mortality from acute myocardial infarction irrespective of the patient's age, sex, blood pressure, and previous history of myocardial infarction or diabetes. ${ }^{1}$ Maximum benefit, however, is seen in those patients treated within 4-6 hours of their symptoms starting. Patients do not always seek medical help soon enough, and this accounts for much of the delay in receiving thrombolytic treatment, but important delays also occur in hospital. These are not related to the route by which the patient is admitted to hospital and vary widely between hospitals. ${ }^{2}$

Although accident and emergency departments are in an important position to minimise any delay in giving thrombolytic treatment, a recent questionnaire study of junior hospital doctors in Scotland showed that thrombolysis is rarely given in accident and emergency departments there. ${ }^{3}$ We surveyed consultants in accident and emergency departments in the United Kingdom to establish current practice and to determine how many consultants felt that thrombolytic treatment should be given routinely in their department.

\section{Methods and results}

We sent a questionnaire to consultants in the 295 accident and emergency departments identified from the directory of emergency and special care units in the United Kingdom ${ }^{4}$; all were sent a reminder letter after 3 weeks. All responses were anonymous. The questionnaire asked whether thrombolytic treatment was currently given in the accident and emergency department; if the hospital had formal policies for giving priority to patients who would benefit from this treatment ("fast tracking") or written policies on giving thrombolytic treatment in accident and emergency departments; about the time lag between a patient's arrival in hospital and starting thrombolytic treatment ("door to needle" time); and whether the consultant thought thrombolysis should be given routinely in the department.

Altogether 264 questionnaires were returned (response rate 89\%). Two hundred and thirteen (81\%) were from consultants in district general hospitals, 44 $(17 \%)$ from teaching hospitals, and $7(2 \%)$ from others (general practitioner hospitals, minor injury units, and military hospitals). Fifty nine (22\%) of the hospitals had no fast track policy, and only 167 (63\%) had any form of written policy on giving thrombolytic treatment in the accident and emergency department (table).

Consultants' responses (number (\%)) to questions on thrombolytic treatment in accident and emergency departments in the United Kingdom

\begin{tabular}{lcccc} 
& Routinely & Occasionally & Rarely & Never \\
\hline $\begin{array}{l}\text { Is thrombolysis given in your department? } \\
(n=264)\end{array}$ & $93(35)$ & $67(25)$ & $47(18)$ & $57(22)$ \\
$\begin{array}{l}\text { Should thrombolysis be given in accident and } \\
\text { emergency? }(n=261)\end{array}$ & $152(58)$ & $93(36)$ & - & $16(6)$ \\
\hline
\end{tabular}

Although only $93(35 \%)$ accident and emergency departments gave thrombolysis routinely, $152(58 \%)$ of the respondents believed that this should be the case. Thrombolytic treatment was given routinely in accident and emergency departments in $61 \%$ of teaching hospitals but in only $31 \%$ of district general hospitals. One third of respondents did not know what the "door to needle" time was in their hospital. Where thrombolysis was given routinely in accident and emergency, the mean (SD) door to needle time was 33 (12) minutes, but when this treatment was given elsewhere in the hospital the time lag was 44 (26) minutes $(\mathrm{P}=0.004, t$ test $)$.

\section{Comment}

We asked consultants whether patients with acute myocardial infarction were given thrombolytic treatment routinely in accident and emergency departments, and if not whether this should be the practice. In a study such as this, clinical practice and questionnaire responses may differ, resulting in bias. Nevertheless, these responses show that many hospitals do not have fast track policies or give thrombolysis routinely in accident and emergency departments, although these measures would reduce the door to needle time. Most respondents believed that patients with acute myocardial infarction admitted to an accident and emergency department should receive thrombolytic treatment there, but only a third of departments currently give this treatment.

The considerable reduction in the door to needle time when thrombolysis is given routinely in accident and emergency departments suggests that some hospitals could reduce delays by adopting this procedure. Further research and the formulation of evidence based guidelines are needed.

We thank all those consultants who completed the questionnaire.

Contributors: $\mathrm{SH}$ was responsible for the initial idea, designing the questionnaire, and writing the papter, and he will act as guarantor. $\mathrm{DB}$ was responsible for designing the questionnaire and collecting and analysing the data, and he contributed to the writing of the paper. LS was responsible for designing the questionnaire and collecting the data, and she contributed to the writing of the paper. WSH was responsible for interpreting the findings, editing the paper, and overall supervision of the project.

Funding: None.

Conflict of interest: None.

1 Fibrinolytic Therapy Trialists' (FTT) Collaborative Group. Indications for fibrinolytic therapy in suspected acute myocardial infarction: collaborative overview of early mortality and major morbidity results from all randomised trials of more than 1000 patients. Lancet 1994;343:311-22.

2 Birkhead JS on behalf of Joint Audit Committee of the British Cardiac Society and Cardiology Committee of the Royal College of Physicians of London. Time delays in provision of thrombolytic treatment in six district hospitals. BMJ 1992;305:445-8.

3 Hood S, Birnie D, Curzio JL, Hillis WS. Wide variation in the use of thrombolytic therapy among junior doctors in south and central Scotland. Health Bull 1996;54:131-9.

4 UK Directory of Emergency and Special Care Units. Cambridge: CMA Medical Data, 1997.

(Accepted 8 April 1997) 